\title{
Patterns of Migraine in Postmenopausal Women: A Systematic Review
}

This article was published in the following Dove Press journal:

Neuropsychiatric Disease and Treatment

\author{
Raffaele Ornello (D) \\ Valeria Caponnetto' \\ Ilaria Frattale (iD ${ }^{2}$ \\ Simona Saccol \\ 'Department of Applied Clinical Sciences \\ and Biotechnology, University of L'Aquila, \\ L'Aquila, Italy; ${ }^{2}$ Child Neurology and \\ Psychiatry Unit, Systems Medicine \\ Department, Tor Vergata University, \\ Rome, Italy
}

Introduction: Migraine prevalence is higher in fertile than in postmenopausal women. However, few literature data are available on the prevalence and characteristics of migraine after the menopause and on the effect of hormones in postmenopausal women with migraine. Methods: We performed a systematic literature review of studies available on Scopus and Web of Science from the beginning off indexing until October 18th, 2020. We included both randomized trials and observational studies.

Results: We included 12 papers, six of which assessed the prevalence and characteristics of migraine in postmenopausal women, while the other six assessed the effect of hormones on migraine after the menopause. One of the studies was a randomized trial, while the remaining 11 were observational studies. Ten studies were clinic-based, while the remaining two were population-based. Studies assessing the prevalence and characteristics of migraine after the menopause reported inconsistent findings; in studies performed in headache clinics, likely affected by selection bias towards the most severe cases, a relevant proportion of women reported migraine worsening after the menopause. Studies assessing the effect of hormones on migraine after the menopause showed that postmenopausal hormone replacement therapy was invariably associated with migraine worsening, if containing estrogen.

Conclusion: Our systematic review showed that migraine could be a relevant health problem in postmenopausal women, mostly in headache clinics. However, the available studies allow a limited assessment of the prevalence and characteristics of postmenopausal migraine. Further large studies are needed to better determine the burden of migraine after the menopause according to migraine characteristics and the impact of hormonal treatments.

Keywords: migraine, menopause, systematic review, hormone replacement treatment

\section{Introduction}

Migraine is a primary headache disorder affecting $14 \%$ of the human population. ${ }^{1}$ Migraine is particularly common among subjects aged $<50$ years, among whom it represents the first cause of disability and of years of life lost. ${ }^{2}$ Migraine prevalence and features are influenced by female sex hormones, and mostly fluctuations in estrogen levels. $^{3-5}$ Therefore, migraine is common in women in their fertile period, which is characterized by cyclical variations in estrogen levels, ${ }^{6}$ and during the menopausal transition, when high estrogen fluctuations may occur, while the postmenopausal period, characterized by stable low estrogen levels, is usually associated with an improvement or even cessation of migraine symptoms. ${ }^{7}$ Despite that, postmenopausal migraine can have an important burden to society due to the high prevalence of the disease. The study of postmenopausal migraine is also interesting
Correspondence: Raffaele Ornello Department of Applied Clinical Sciences and Biotechnology, University of L'Aquila, L'Aquila, Italy

Tel +393288756932

Fax +39086243356I

Email raffaele.ornello@gmail.com
Neuropsychiatric Disease and Treatment 2021:17 859-87| 
to investigate the effects of female sex hormone changes, including hormonal manipulation strategies, on migraine.

In the present systematic review, we aimed to present data about migraine patterns in postmenopausal women, with special attention to the role of sex hormones.

\section{Methods}

We conducted a systematic review following the 'Cochrane Handbook for Systematic Reviews of Interventions', Version 5.1.0 ${ }^{8}$ for conduction and the 'Preferred Reporting Items for Systematic Reviews and Meta-Analyses' (PRISMA) checklist for reporting. ${ }^{9}$

\section{Search Strategy}

We combined the keywords "migraine", "episodic migraine", "chronic migraine", CM, "migraine disorder", "migraine with aura", "migraine without aura" and "postmenopause" (with all possible synonyms) for the search strategy. We launched search strings on Scopus and Web of Science on October 18th, 2020. We limited search results to journal articles and surveys published in English or Italian. Complete search strings are reported in Supplemental Table 1. Retrieved references were managed with Endnote free Web.

\section{Study Selection}

Studies were screened for eligibility and inclusion analyzing title/abstract and full texts, respectively, by two raters (IF, VC) independently. To be evaluated in full texts, titles and abstracts of retrieved references had to include adult patients with migraine. Moreover, in this phase, systematic reviews, book chapters, proceedings, case reports, letters, and editorials were excluded. In order to be included in the systematic review, studies had to: a) be available in full text; b) be published on peer-reviewed journals in Italian or English; c) be primary; d) be conducted on migraine patients; e) be conducted on postmenopausal migraine women; f) report details about migraine patterns in postmenopausal women. Both in the eligibility and inclusion stage, the agreement among the judgements of the raters (inter-rater reliability) was estimated with the Krippendorff's alpha coefficient $(\alpha)$ ranging from 0 (totally disagree) to 1 (totally agree). ${ }^{10}$ Any disagreement between the raters was resolved by discussion among all the Authors until consensus was reached.

We evaluated the risk of bias of included studies with the "Downs and Black instrument", ${ }^{11}$ after having modified it as needed. For each study, a total standardized score was computed to provide an overall evaluation of the risk of bias. Risk of bias evaluation was performed independently by two raters ( $\mathrm{VC}, \mathrm{RO}$ ). Any disagreement between the raters was resolved by discussion among all Authors until consensus was reached.

\section{Data Collection and Analysis}

Data extraction was performed through an ad hoc electronic spreadsheet of Microsoft Excel for Windows by the first author who extracted the following data: first author, publication year, study methods (ie, study design, number of involved patients, age range of the sample, and number of postmenopausal migraine women), details about migraine patterns in postmenopausal migraine women (ie, migraine prevalence, migraine change according to hormonal changes). We narratively synthetized studies methods and details about migraine patterns in postmenopausal migraine women. Due to the high heterogeneity of the reviewed studies, we did not perform any formal metaanalysis.

\section{Results \\ Search Results}

The electronic searches in the scientific databases identified 1296 potentially relevant records: after removing duplicates, we assessed for eligibility titles and abstracts of 1265 references. Afterwards, we evaluated 50 full-text articles and included 12 studies $^{12-23}$ in the systematic review (Figure 1). In the eligibility and inclusion stage, the agreement among the judgements of the authors (Krippendorff's alpha coefficient, $\alpha$ ) was 0.90 and 0.96 , respectively.

All the included studies had medium to high quality according to the Downs and Black's checklist (Table 1).

\section{Migraine Prevalence and Characteristics in Postmenopausal Women}

Six studies assessed migraine prevalence and characteristics in postmenopausal women (Table 2) ${ }^{12,13,18,20-22}$ Four studies were performed in headache clinics, ${ }^{12,13,18,21}$ one in a gynecology clinic, ${ }^{20}$ and one was a survey in the general population. ${ }^{22}$

Two of the studies performed in headache clinics found that the postmenopausal period was associated with less frequent and/or less severe migraine in more than half of women, ${ }^{12}$ while another study performed in a headache clinic found that most women reported migraine 


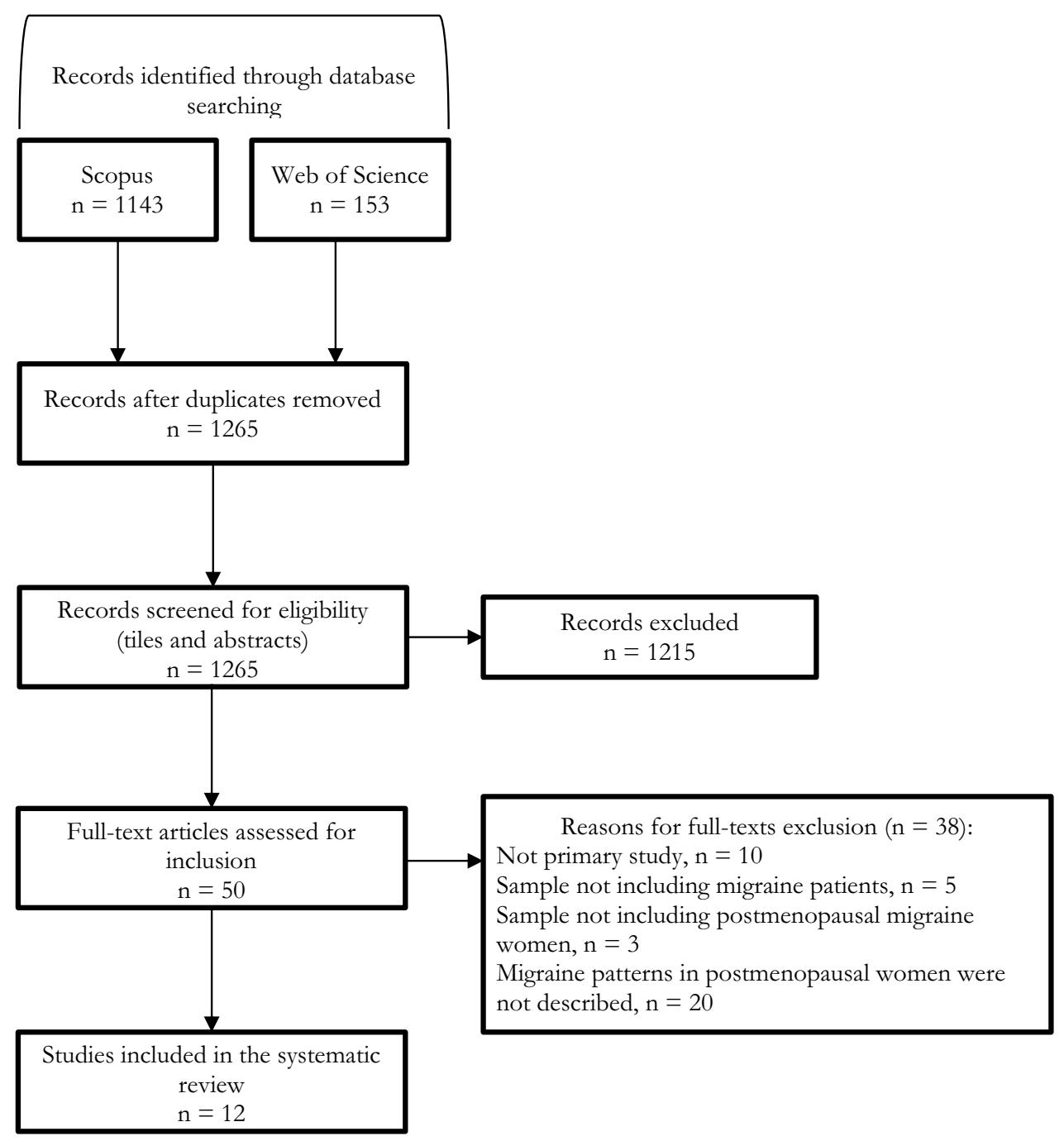

Figure I Flowchart of study selection.

worsening after the menopause; ${ }^{13}$ in that same study, migraine worsened in all women with surgical menopause, while the two women reporting an improvement in their migraine had undergone natural menopause. ${ }^{13}$ Notably, one study performed in a headache clinic found that eight $(17.0 \%)$ of 47 postmenopausal women reported the onset of their migraine in the postmenopausal period. ${ }^{12}$

The study performed in a gynecology clinic found that migraine was present in $14.7 \%$ of women in the postmenopausal period; most of those women had a mild migraine-related disability; however, postmenopausal women with migraine had a higher prevalence of menopausal and depressive symptoms compared with those without migraine. $^{20}$

The study performed in the general population showed that migraine frequency, duration, and associated symptoms were higher in women than in men even after the menopause; that study did not directly compare postmenopausal with pre-menopausal women. ${ }^{22}$

In all the reviewed studies, no information was available regarding the difference between migraine with and without aura.

\section{The Role of Sex Hormones in Postmenopausal Women with Migraine}

Overall, six studies assessed the effect of hormones on postmenopausal migraine (Table 3). ${ }^{14-17,19,23}$ One study was a randomized controlled trial of hormonal replacement therapy (HRT), ${ }^{16}$ while four studies reported observational data on the effects of different HRT regimens, ${ }^{14,15,17,19}$ only one study reported the levels of 


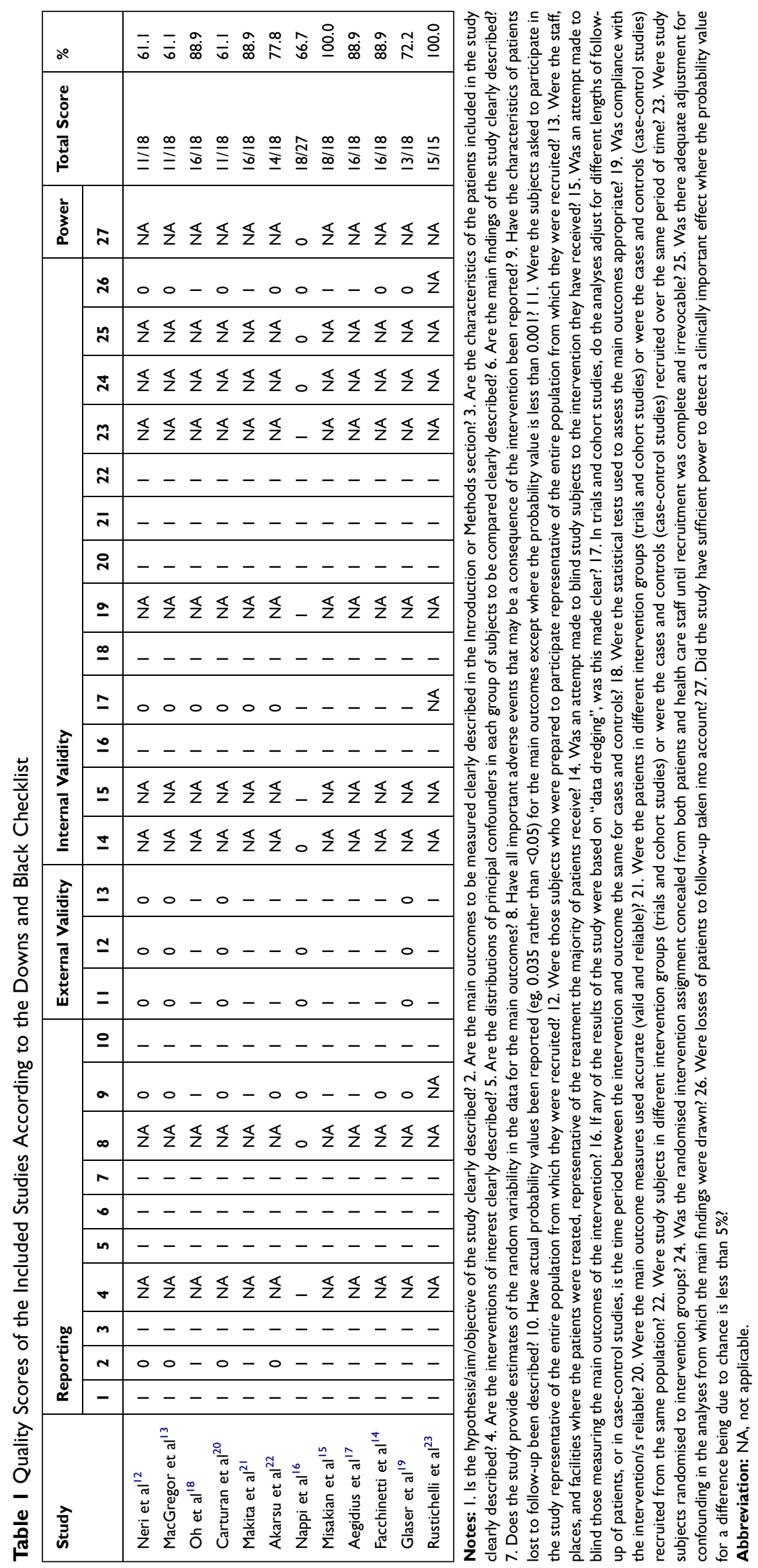




\begin{tabular}{|c|c|c|c|c|}
\hline 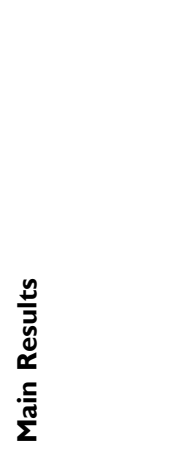 & 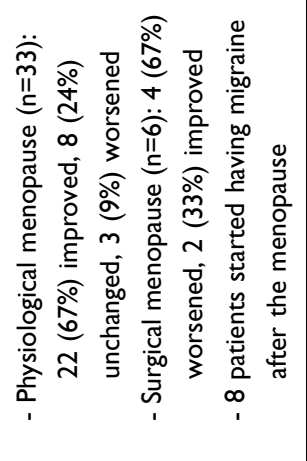 & 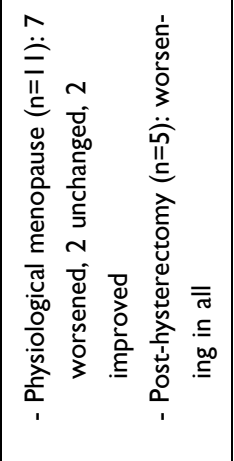 & 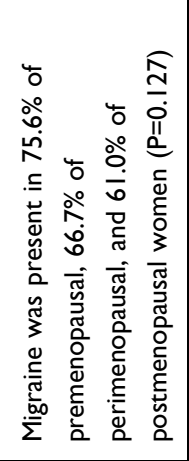 & 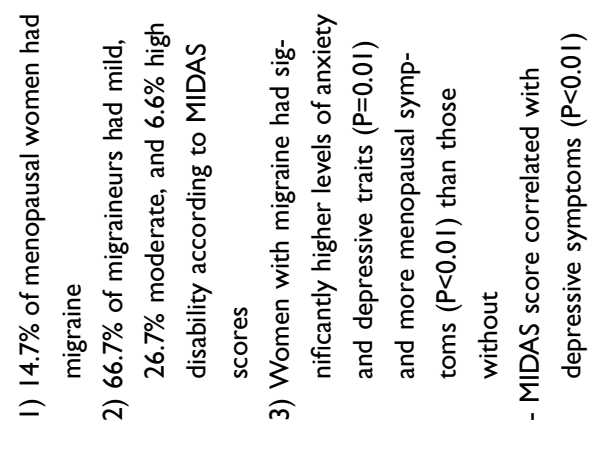 \\
\hline 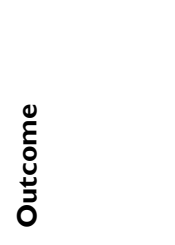 & 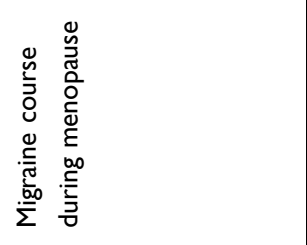 & 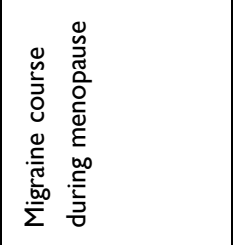 & 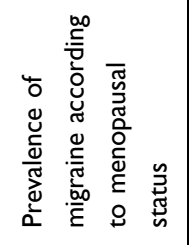 & 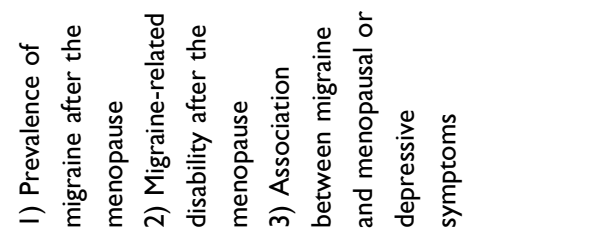 \\
\hline 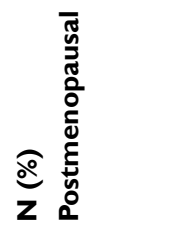 & $\begin{array}{l}\stackrel{0}{\dot{0}} \\
\stackrel{0}{=} \\
\text { f }\end{array}$ & $\begin{array}{l}\underset{0}{0} \\
\stackrel{\dot{\omega}}{0} \\
\underline{0}\end{array}$ & 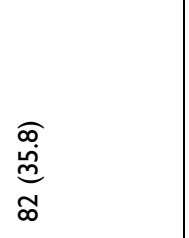 & $\begin{array}{l}\stackrel{o}{\dot{0}} \\
\stackrel{0}{0} \\
\underline{0}\end{array}$ \\
\hline 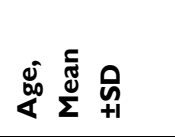 & $\stackrel{\alpha}{z}$ & 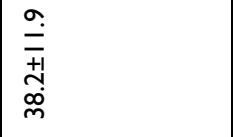 & 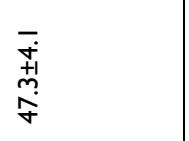 & $\begin{array}{l}\overline{\dot{p}} \\
\stackrel{+}{N} \\
\text { n }\end{array}$ \\
\hline 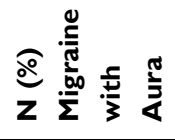 & 0 & $\begin{array}{l}\underset{\mathrm{d}}{\mathrm{d}} \\
\text { స }\end{array}$ & $\stackrel{\mathscr{c}}{Z}$ & $\stackrel{\alpha}{z}$ \\
\hline $\mathbf{z}$ & fे & $\underline{\underline{B}}$ & సి & 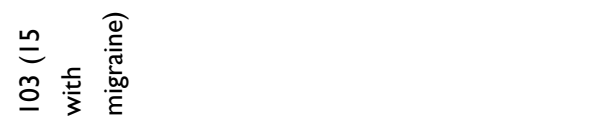 \\
\hline 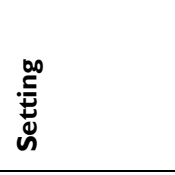 & 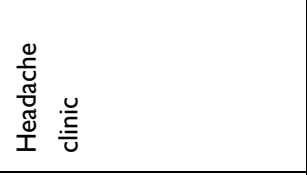 & 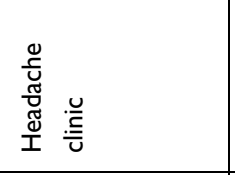 & 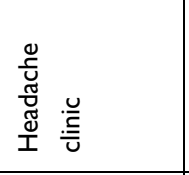 & 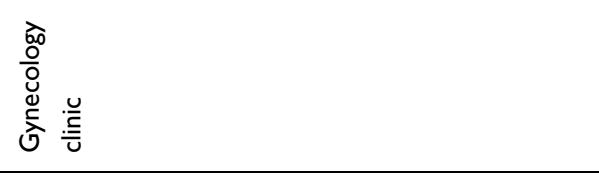 \\
\hline 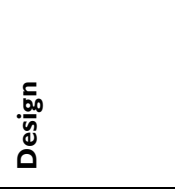 & 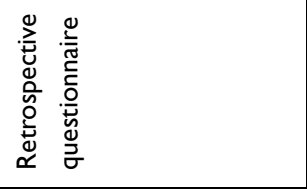 & 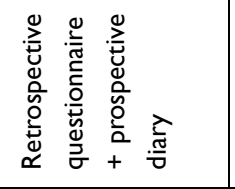 & 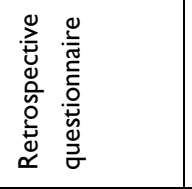 & 它 \\
\hline نे & 촡 & 弚 & 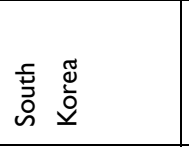 & $\begin{array}{l}\overline{\bar{N}} \\
\bar{\Phi} \\
\bar{D}\end{array}$ \\
\hline 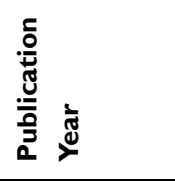 & $\stackrel{m}{\sigma}$ & $\underline{\sigma}$ & ํํำ & 음 \\
\hline 啇 & 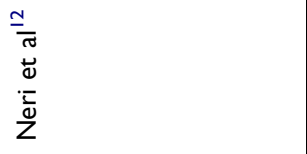 & 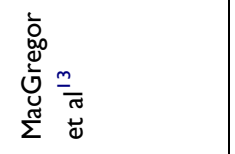 & $\begin{array}{l}\frac{\infty}{\bar{\sigma}} \\
\stackrel{0}{0} \\
\frac{\tau}{0}\end{array}$ & 施 \\
\hline
\end{tabular}




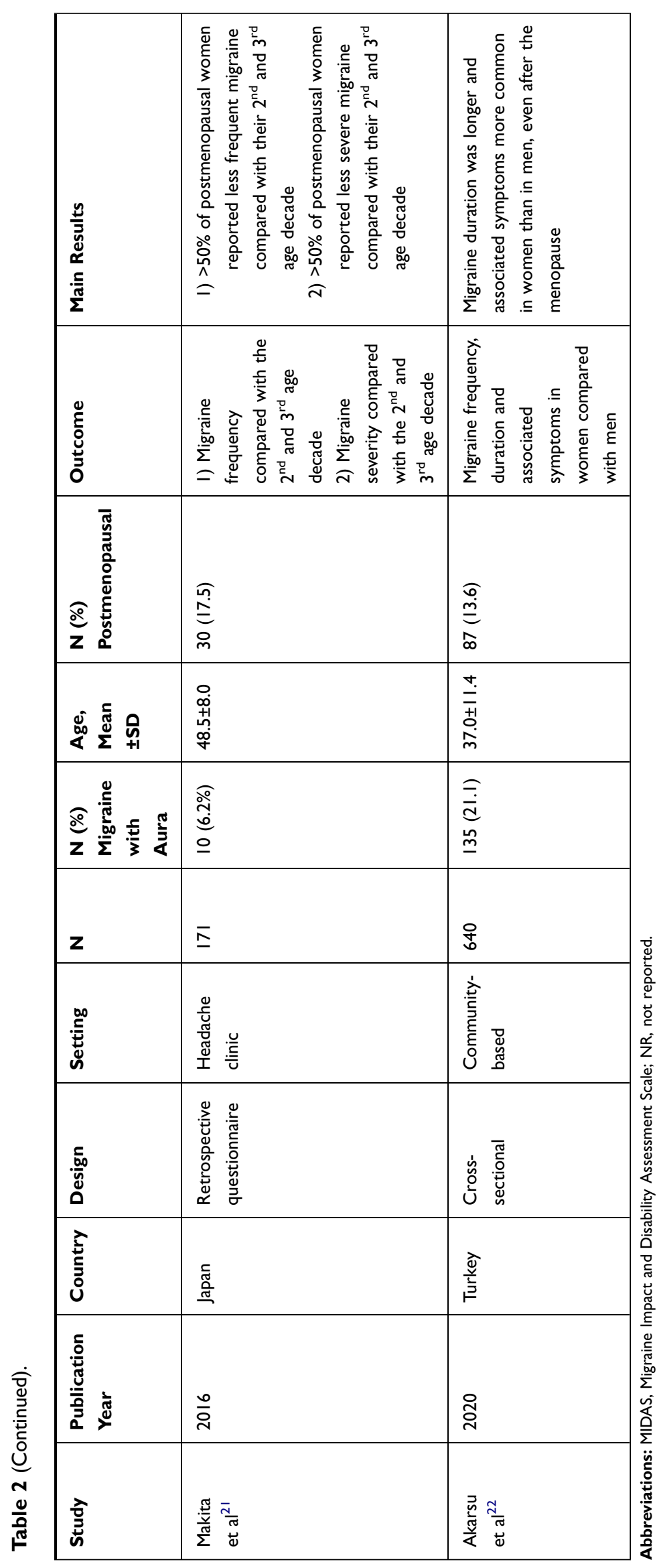




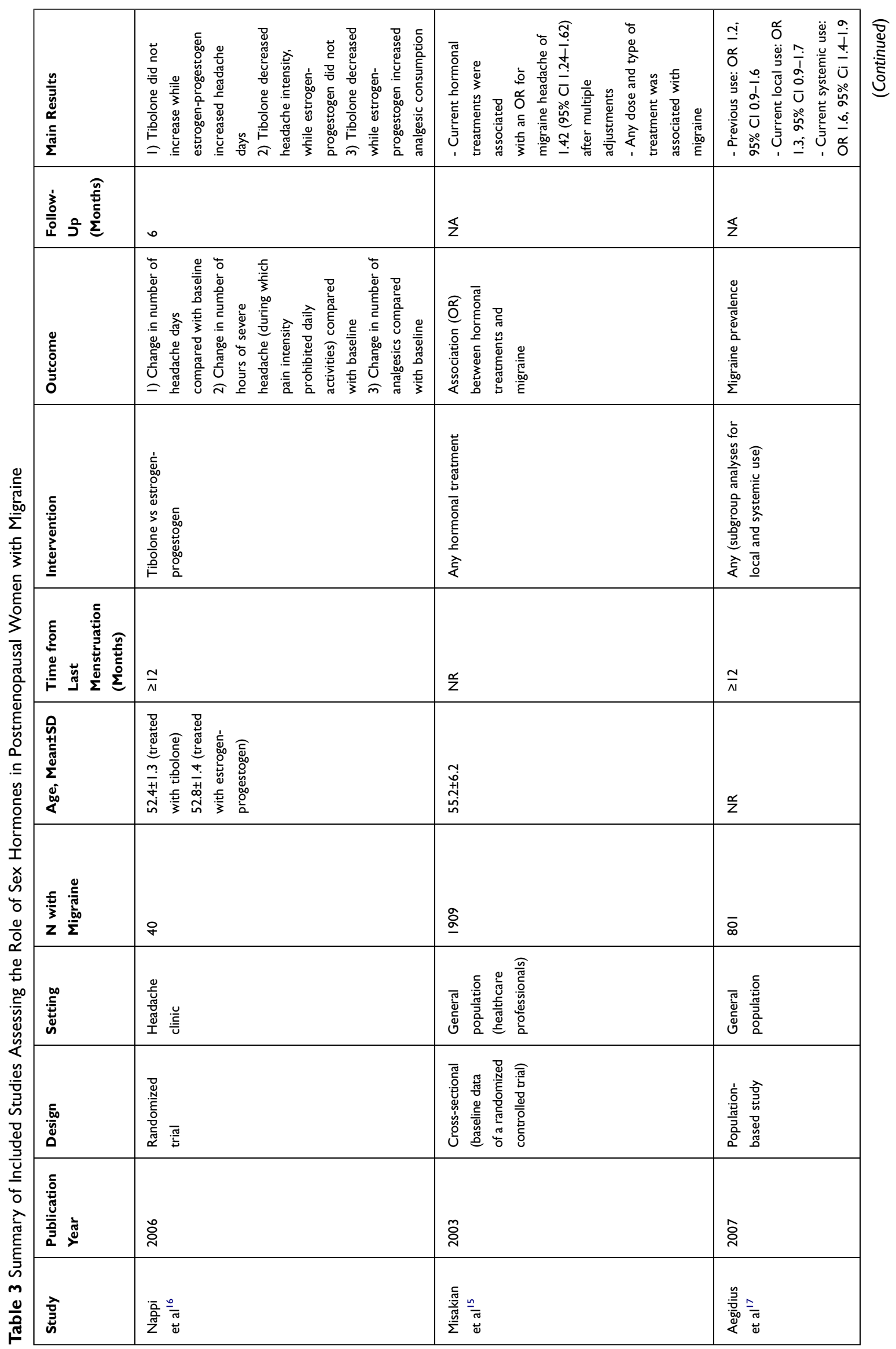




\begin{tabular}{|c|c|c|}
\hline & 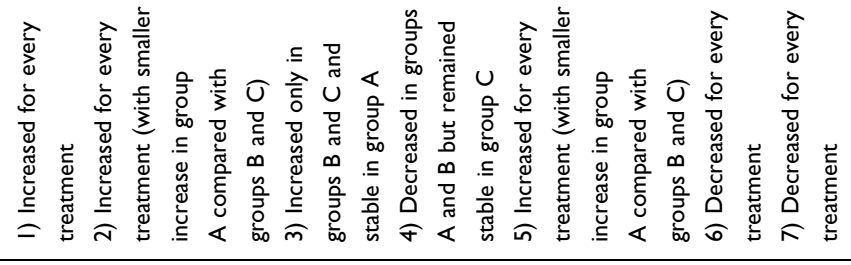 & 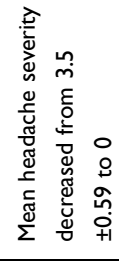 \\
\hline 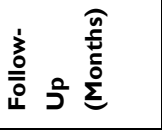 & $\stackrel{\circ}{\ddot{m}}$ & $m$ \\
\hline & 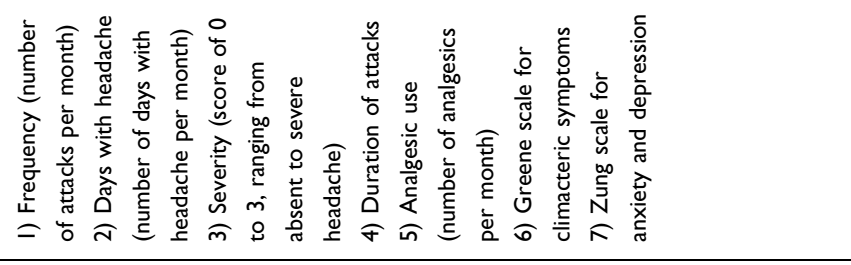 & 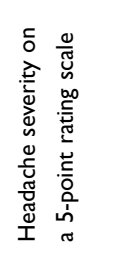 \\
\hline & 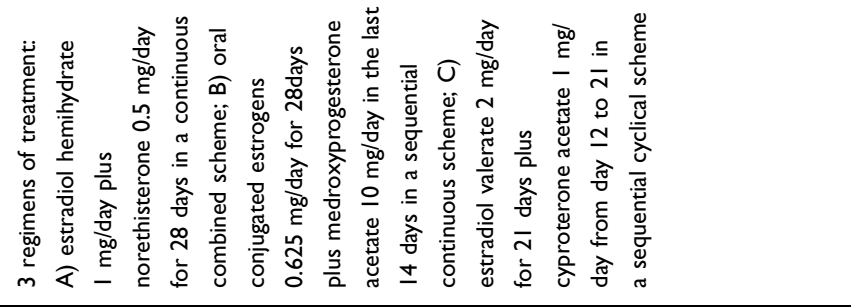 & 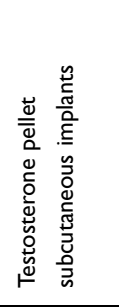 \\
\hline 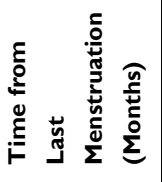 & 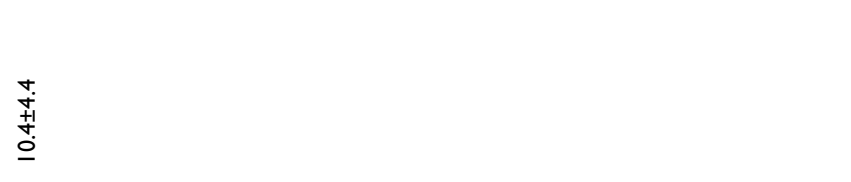 & $\stackrel{\alpha}{z}$ \\
\hline & $\frac{\frac{\sigma}{+1}}{\frac{\dot{m}}{n}}$ & 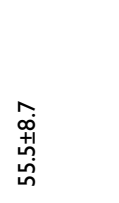 \\
\hline 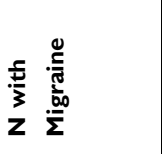 & $\stackrel{\infty}{\infty}$ & $=$ \\
\hline 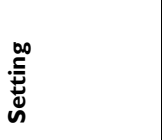 & 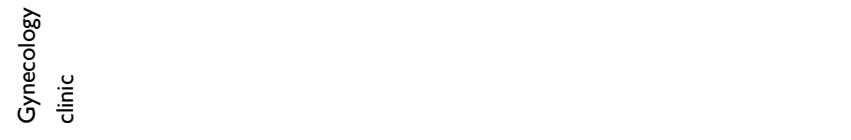 & 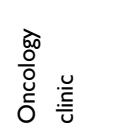 \\
\hline $\begin{array}{l}\frac{5}{50} \\
\overline{0} \\
0\end{array}$ & 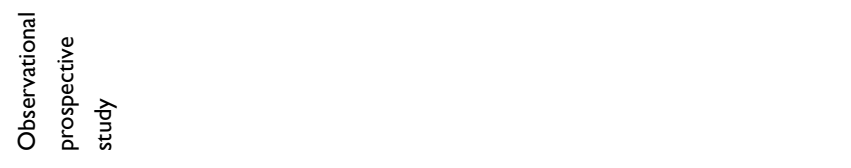 & 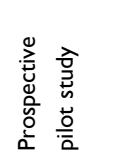 \\
\hline 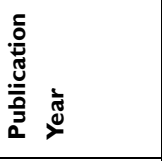 & ర్లి & $\stackrel{\sim}{\circ}$ \\
\hline 六 & 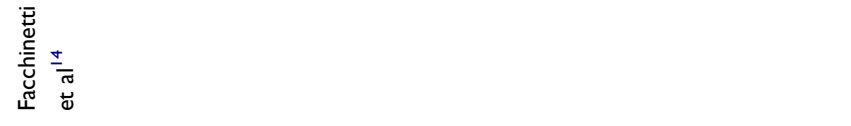 & 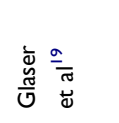 \\
\hline
\end{tabular}




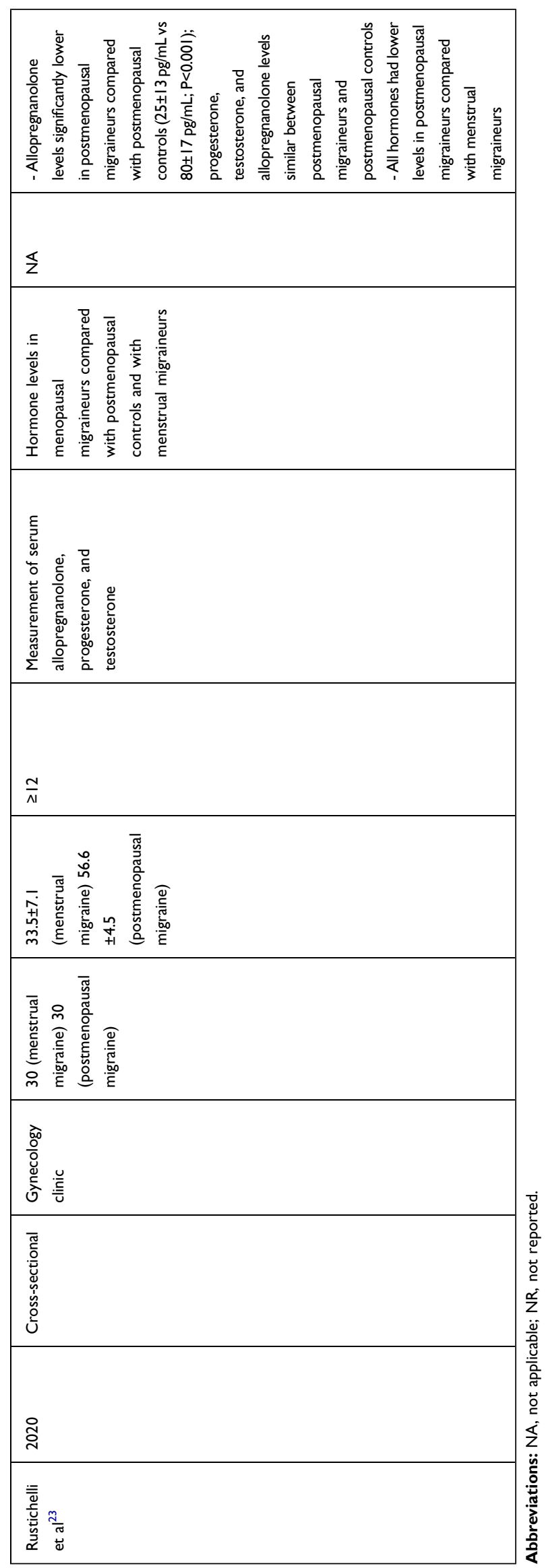


natural sex hormones in postmenopausal women with migraine. $^{23}$

The randomized controlled trial compared tibolone, a non-estrogen synthetic steroid, with conventional estrogen-progestogen over a 6-month follow-up in postmenopausal women with migraine without aura; this study found that tibolone did not increase headache days compared with baseline, while decreasing headache intensity and analgesic consumption; on the contrary, conventional estrogen-progestin treatment increased headache days and analgesic consumption, while not decreasing headache intensity. $^{16}$

Two cross-sectional studies assessed the association between the use of postmenopausal HRT and migraine. ${ }^{15,17}$ One study found that any type of HRT was associated with migraine, ${ }^{15}$ the other study found that current and systemic hormonal treatments were associated with increased odds for migraine, while previous and local HRT were not associated with an increased odds of having migraine. ${ }^{17}$ A prospective observational study assessed the effect of three different HRT regimens - a continuous combined, a sequential continuous, and a sequential cyclical scheme - on postmenopausal migraine. ${ }^{14}$ All regimens worsened migraine frequency, duration, and analgesic use, while improving climacteric symptoms; however, the continuous combined scheme had the lowest overall impact on migraine, with the smallest increase in headache days and analgesic use and stable headache severity; on the contrary, the sequential cyclical scheme had the highest impact on migraine worsening. ${ }^{14}$

A pilot study observed the effect of testosterone implants in women with breast cancer; that study found that all postmenopausal women with migraine treated with subcutaneous implants of testosterone had a cessation of their migraine within 3 months of follow-up. ${ }^{19}$

One study assessed the natural serum levels of different sex hormones in menopausal women according to their migraine status; the levels of allopregnanolone were significantly lower in postmenopausal women with migraine compared with those without migraine, while the levels of other sex hormones did not differ between the two groups. $^{23}$

\section{Discussion}

We summarized the results and potential implications of our review in Figure 2. Our systematic literature search showed that the available studies on the patterns of migraine in postmenopausal women often provide incomplete information and are affected by potential selection bias. The burden and characteristics of migraine are
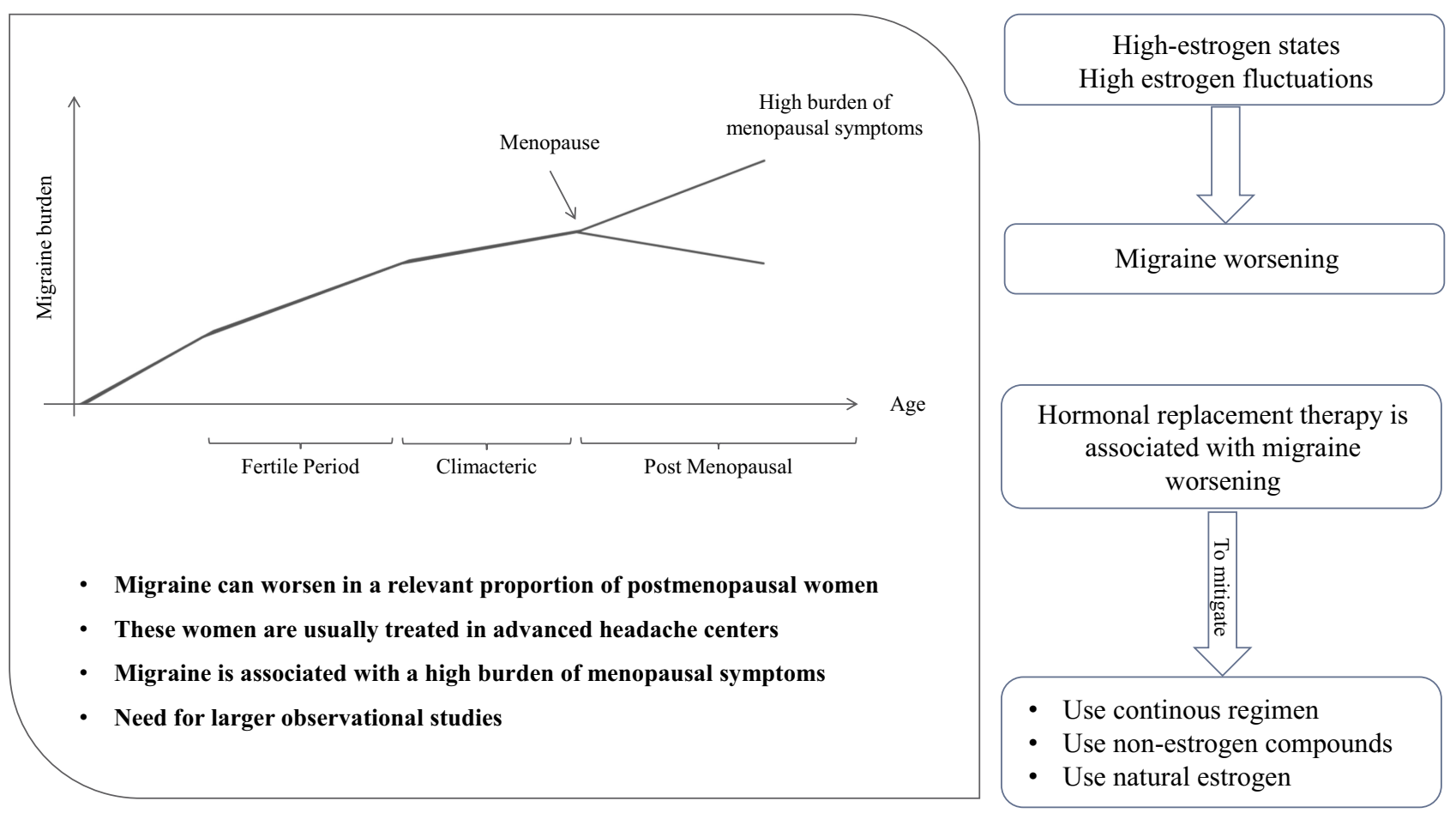

Figure 2 Summary of findings and implications of the present review. 
difficult to determine in women several years after the menopause. The available literature data are mostly focused on the menopausal transition, a period of high susceptibility to migraine due to fluctuations in female sex hormones, while few data are available on the course of migraine years after the menopause. It is commonly accepted that migraine ameliorates after the menopause. ${ }^{7}$ However, the reviewed studies showed that migraine worsening can be found in a relevant proportion of postmenopausal women. ${ }^{12,13}$ Most of the available data are from retrospective studies performed in headache clinics selecting the most severe cases of migraine and are therefore not representative of the general population. Nevertheless, those data are important as they underline that headache centers usually treat a high number of postmenopausal women with migraine. Our review also pointed out that postmenopausal migraine can be a relevant issue for gynecologists, as migraine had a high prevalence (about 15\%) in women attending a menopause clinic and was associated with a high burden of menopausal symptoms. ${ }^{20}$ Collaboration between headache physicians and gynecologists can be useful to assess the real burden of migraine in postmenopausal women, especially several years after the menopause onset, as solid literature data are lacking in this field.

The potential migraine worsening soon after the menopause can be explained by "estrogen withdrawal", ie, rapid falls in estrogen levels. ${ }^{24,25}$ This is the same mechanism which also explains menstrual migraine. ${ }^{3}$ The same mechanism can also explain the association between surgical menopause and migraine worsening, ${ }^{13}$ surgical menopause is indeed associated with a sudden decrease in estrogen levels due to the excision of estrogen-producing organs, therefore leading to worsening of migraine symptoms. However, our literature search did not find any longterm data confirming whether the trend to migraine worsening persists over time in postmenopausal women. Further multicenter studies are needed to assess the epidemiology of postmenopausal migraine in both headache centers and in the general population. Those studies should consider variables such as migraine frequency, duration, and the presence of aura, to help predicting the course of migraine years after the menopause.

The role of male and female sex hormones in postmenopausal migraine is understudied. ${ }^{26}$ Most studies refer to HRT, which is usually prescribed in women with menopausal symptoms and can be considered safe within 10 years after the menopause. ${ }^{27}$ The available literature data unanimously suggest that any estrogen-progestogen combination used for HRT is associated with migraine worsening. ${ }^{14,16}$ Several strategies might be adopted to mitigate the HRT-related migraine worsening in postmenopausal women. The first strategy, in accordance with the estrogen withdrawal theory, is to use continuous regimens, so to avoid estrogen withdrawal and the consequent increased susceptibility to migraine; a continuous HRT strategy showed less impact on migraine compared with cyclic strategies in an observational study. ${ }^{14}$ Another strategy to avoid migraine worsening is using non-estrogen compounds for HRT; indeed, tibolone effectively decreased migraine severity and analgesic consumption. ${ }^{16}$ It has been suggested that natural estrogens can have a decreased impact on migraine compared with synthetic estrogens. ${ }^{28}$ However, our review did not find epidemiological data to confirm this hypothesis in postmenopausal women. The included studies did not give enough data about the course of migraine with aura compared with migraine without aura under HRT. However, it should be noted that migraine with aura is associated with an increased risk of ischemic stroke and other vascular conditions that is further amplified by exogenous hormone use. ${ }^{29-31}$ For that reason, HRT is usually contraindicated in women with migraine with aura, ${ }^{32,33}$ which makes it difficult to assess the safety of HRT in women with that condition.

According to the findings of our review, progestin compounds can also have a role in the hormonal management of postmenopausal migraine. A cross-sectional study included in our review found that women with migraine had similar estrogen levels than those without migraine, while having lower levels of allopregnanolone, a progestin compound. ${ }^{23}$ Progestin hormones are implied in the negative modulation of cortical excitability and can therefore help reducing the susceptibility to migraine. However, we did not find any interventional studies assessing the effect of progestins on postmenopausal migraine.

Male sex hormones can influence the burden of migraine after the menopause. As shown by a study, testosterone implants aborted migraine in all treated postmenopausal women with breast cancer. ${ }^{19}$ In that study, the anti-migraine effect of testosterone was serendipitous, as testosterone was not prescribed as HRT. Future large studies performed in women representative of the general population might assess the safety and efficacy of exogenous male sex hormones in decreasing the burden of migraine after the menopause. 
Our systematic review method ensured the validity and reproducibility of our literature search. However, our review has several limitations related to the characteristics of included studies. We found heterogeneous studies, with different designs and aims, whose results could not be compared with each other. Therefore, we limited our review to a narrative synthesis, without the possibility of grouping studies according to the topics. Besides, despite a medium to high quality according to a validated checklist (Table 1), the studies included a low number of postmenopausal women with migraine, which cannot rule out the presence of selection bias.

\section{Conclusions}

Our systematic review showed that there are few available data on the prevalence and characteristics of migraine after the menopause. The population of postmenopausal women with migraine is poorly considered by current literature despite representing a relevant proportion of patients referring to headache centers. Besides, the effect of hormonal treatments years after the menopause is yet to be extensively explored in women with migraine. Further well-designed and powered observational studies are needed to provide a detailed picture of postmenopausal migraineurs, their characteristics, burden of disease, health resource use, and the effect of different hormonal treatments.

\section{Disclosure}

Dr Raffaele Ornello reports non-financial support from Allergan, personal fees from Teva, outside the submitted work; Dr Valeria Caponnetto reports personal fees from Novartis, outside the submitted work; Prof. Dr. Simona Sacco reports grants, personal fees, non-financial support from Allergan-Abbvie, grants from Novartis, non-financial support from Abbott, outside the submitted work. The authors report no other conflicts of interest in this work.

\section{References}

1. GBD 2016 Headache Collaborators. Global, regional, and national burden of migraine and tension-type headache, 1990-2016: a systematic analysis for the Global Burden of Disease Study 2016. Lancet Neurol. 2018;17(11):954-976. doi:10.1016/S1474-4422(18) 30322-3.

2. Steiner TJ, Stovner LJ, Vos T, Jensen R, Katsarava Z. Migraine is first cause of disability in under 50s: will health politicians now take notice? J Headache Pain. 2018;19(1):17. doi:10.1186/s10194-018-0846-2

3. MacGregor EA, Frith A, Ellis J, Aspinall L, Hackshaw A. Incidence of migraine relative to menstrual cycle phases of rising and falling estrogen. Neurology. 2006;67(12):2154-2158. doi:10.1212/01.wnl.00 00233888.18228 .19
4. Sacco S, Merki-Feld GS, Ægidius KL, et al. Effect of exogenous estrogens and progestogens on the course of migraine during reproductive age: a consensus statement by the European Headache Federation (EHF) and the European Society of Contraception and Reproductive Health (ESCRH). J Headache Pain. 2018;19(1):76. doi:10.1186/s10194-018-0896-5

5. Vetvik KG, MacGregor EA. Sex differences in the epidemiology, clinical features, and pathophysiology of migraine. Lancet Neurol. 2017;16(1):76-87. doi:10.1016/S1474-4422(16)30293-9

6. Sacco S, Ricci S, Degan D, Carolei A. Migraine in women: the role of hormones and their impact on vascular diseases. $J$ Headache Pain. 2012;13(3):177-189. doi:10.1007/s10194-012-0424-y

7. Ripa P, Ornello R, Degan D, et al. Migraine in menopausal women: a systematic review. Int $J$ Womens Health. 2015;7:773-782. doi:10. 2147/IJWH.S70073

8. Higgins J, Thomas J The Cochrane Handbook for Systematic Reviews of Interventions. Available from: https://training.cochrane. org/handbook/current.

9. Liberati A, Altman DG, Tetzlaff J, et al. The PRISMA statement for reporting systematic reviews and meta-analyses of studies that evaluate healthcare interventions: explanation and elaboration. $B M J$. 2009;339(jul21 1):b2700. doi:10.1136/bmj.b2700

10. Krippendorff K. Computing Krippendorff's alpha reliability. Available from: https://repository.upenn.edu/cgi/viewcontent.cgi?arti $\mathrm{cle}=1043 \&$ context $=$ asc _papers. Accessed December 31, 2020.

11. Downs SH, Black N. The feasibility of creating a checklist for the assessment of the methodological quality both of randomised and non-randomised studies of health care interventions. $J$ Epidemiol Community Health. 1998;52(6):377-384. doi:10.1136/jech.52.6. 377

12. Neri I, Granella F, Nappi R, Manzoni GC, Facchinetti F, Genazzani AR. Characteristics of headache at menopause: a clinico-epidemiologic study. Maturitas. 1993;17(1):31-37. doi:10. 1016/0378-5122(93)90121-w

13. MacGregor EA. Menstruation, sex hormones, and migraine. Neurol Clin. 1997;15(1):125-141. doi:10.1016/s0733-8619(05)70299-1

14. Facchinetti F, Nappi RE, Tirelli A, Polatti F, Nappi G, Sances G. Hormone supplementation differently affects migraine in postmenopausal women. Headache. 2002;42(9):924-929. doi:10.1046/j.15264610.2002.02215.x

15. Misakian AL, Langer RD, Bensenor IM, et al. Postmenopausal hormone therapy and migraine headache. $J$ Womens Health (Larchmt). 2003;12(10):1027-1036. doi:10.1089/154099903322643956

16. Nappi RE, Sances G, Sommacal A, et al. Different effects of tibolone and low-dose EPT in the management of postmenopausal women with primary headaches. Menopause. 2006;13(5):818-825. doi:10.10 97/01.gme.0000227399.53192.f5

17. Aegidius KL, Zwart JA, Hagen K, Schei B, Stovner LJ. Hormone replacement therapy and headache prevalence in postmenopausal women. The Head-HUNT study. Eur J Neurol. 2007;14(1):73-78. doi:10.1111/j.1468-1331.2006.01557.x

18. Oh K, Jung KY, Choi JY, Seo WK, Park KW. Headaches in middle-aged women during menopausal transition: a headache clinic-based study. Eur Neurol. 2012;68(2):79-83. doi:10.1159/00 0336838

19. Glaser R, Dimitrakakis C, Trimble N, Martin V. Testosterone pellet implants and migraine headaches: a pilot study. Maturitas. 2012;71 (4):385-388. doi:10.1016/j.maturitas.2012.01.006

20. Carturan P, Scorcine C, Fragoso YD. Migraine in the post-menopausal period is associated with higher levels of mood disorders, disability, and more menopausal symptoms. Arq Neuropsiquiatr. 2016;74(12): 999-1002. doi:10.1590/0004-282X20160153

21. Makita K, Inagaki M, Kitamura S, Tatsuoka Y. Changes in migraine before and after menopause in Japanese climacteric women. Cephalalgia. 2017;37(11):1088-1092. doi:10.1177/033310241665 3234 
22. Akarsu EO, Baykan B, Ertaş M, et al. Sex differences of migraine: results of a nationwide home-based study in Turkey. Noro Psikiyatr Ars. 2020;57(2):126-130. doi:10.29399/npa.23240

23. Rustichelli C, Bellei E, Bergamini S, et al. Serum levels of allopregnanolone, progesterone and testosterone in menstrually-related and postmenopausal migraine: a cross-sectional study. Cephalalgia. 2020;40(12):1355-1362. doi:10.1177/0333102420937742

24. Somerville BW. The role of estradiol withdrawal in the etiology of menstrual migraine. Neurology. 1972;22(4):355-365. doi:10.1212/ wnl.22.4.355

25. MacGregor EA. Oestrogen and attacks of migraine with and without aura. Lancet Neurol. 2004;3(6):354-361. doi:10.1016/S1474-4422 (04)00768-9

26. Delaruelle Z, Ivanova TA, Khan S, et al. Male and female sex hormones in primary headaches. J Headache Pain. 2018;19(1):117. doi:10.1186/s10194-018-0922-7

27. Hamoda H, Panay N, Pedder H, Arya R, Savvas M. The British Menopause Society \& Women's Health Concern 2020 recommendations on hormone replacement therapy in menopausal women. Post Reprod Health. 2020;26(4):181-209. doi:10.1177/20533691 20957514
28. MacGregor EA. Migraine, menopause and hormone replacement therapy. Post Reprod Health. 2018;24(1):11-18. doi:10.1177/205 3369117731172

29. Sacco S, Olivieri L, Bastianello S, Carolei A. Comorbid neuropathologies in migraine. J Headache Pain. 2006;7(4):222-230. doi:10.10 07/s10194-006-0300-8

30. Sacco S, Cerone D, Carolei A. Comorbid neuropathologies in migraine: an update on cerebrovascular and cardiovascular aspects. J Headache Pain. 2008;9(4):237-248. doi:10.1007/s10194-008-0048-4

31. Sacco S, Kurth T. Migraine and the risk for stroke and cardiovascular disease. Curr Cardiol Rep. 2014;16(9):524. doi:10.1007/s11886-0140524-1

32. Sacco S, Merki-Feld GS, Ægidius KL, et al. Hormonal contraceptives and risk of ischemic stroke in women with migraine: a consensus statement from the European Headache Federation (EHF) and the European Society of Contraception and Reproductive Health (ESC). $J$ Headache Pain. 2017;18(1):108. doi:10.1186/s10194-017-0815-1

33. Ornello R, Canonico M, Merki-Feld GS, et al. Migraine, low-dose combined hormonal contraceptives, and ischemic stroke in young women: a systematic review and suggestions for future research. Expert Rev Neurother. 2020:1-5. doi:10.1080/14737175.2020.1730816.
Neuropsychiatric Disease and Treatment

\section{Publish your work in this journal}

Neuropsychiatric Disease and Treatment is an international, peerreviewed journal of clinical therapeutics and pharmacology focusing on concise rapid reporting of clinical or pre-clinical studies on a range of neuropsychiatric and neurological disorders. This journal is indexed on PubMed Central, the 'PsycINFO' database and CAS, and is the official journal of The International Neuropsychiatric Association (INA). The manuscript management system is completely online and includes a very quick and fair peer-review system, which is all easy to use. Visit http://www.dovepress.com/testimonials.php to read real quotes from published authors. 